\title{
FINAL PROJECT SUMMARY FOR DoE/HEP DE-FG02-95ER40911
}

\section{Data Analysis of the LSND for $\pi^{o} \rightarrow \nu \bar{\nu}$ Search}

\author{
A.R. Fazely \\ Southern University and ABM College \\ Baton Rouge,LA 70813 \\ Contact: fazely@feynman.phys.subr.edu
}

The Department of Physics at Southern University participated in the Liquid Scintillator Neutrino Detector experiment (LSND). The grant was provided by the Department of Energy, Office of High energy Physics (DoE/HEP) under the contract number DE-FG0295ER40911.

This report contains the salient features of the activities and achievements obtained through this grant. The total amount of grant for the nine (9) year duration was $\$ 251,000$.

We analyzed both Decay-in-Flight (DIF) neutrino oscillations and $\pi^{0} \rightarrow \nu_{\mu} \bar{\nu}_{\mu}$ decay. In the DIF analysis, we came up with the idea of looking at the charged-current reaction exciting the ground state of ${ }^{12} N$ with a life-time of $15.9 \mathrm{~ms}$. We wrote the section on this search in the LSND "famous" paper, "Results on $\nu_{\mu} \rightarrow \nu_{e}$ Oscillations from Pion Decay in Flight Neutrinos", C. Athanassopoulos, et al.; Phys. Rev. C. 58, 2489, (1998). This analysis was the topic of a 1998 MS thesis for the first graduate, Anthony K. Cochran, of the MS program established in 1996. Tony spend eight months at Los Alamos working on LSND. He was supported by LANL through their Office of Diversity program. Tony is now at Clark-Atlanta University working on his Ph.D.

We also took the lead in analyzing the LSND data for a search for the conventionally forbidden decay of neutral pion to two neutrinos. We wrote a paper on this analysis and this paper was published in Physical Review Letters in March of 2004. "Search for Decay in LSND " L.B. Auerbach, R.L. Burman, D.O. Caldwell, E.D. Church, A.K. Cochran, J.B. Donahue, A.R. Fazely, G.T. Garvey, R.M. Gunasingha, R.L. Imlay, G. Kahrimanis, W.C. Louis, R. Majkic, A. Malik, K.L. McIlhany, W.J. Metcalf, G.B. Mills, D. Rupnik, V.D.

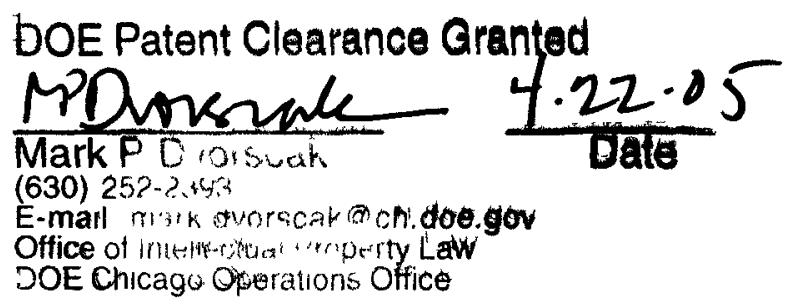




\section{DISCLAIMER}

This report was prepared as an account of work sponsored by an agency of the United States Government. Neither the United States Government nor any agency Thereof, nor any of their employees, makes any warranty, express or implied, or assumes any legal liability or responsibility for the accuracy, completeness, or usefulness of any information, apparatus, product, or process disclosed, or represents that its use would not infringe privately owned rights. Reference herein to any specific commercial product, process, or service by trade name, trademark, manufacturer, or otherwise does not necessarily constitute or imply its endorsement, recommendation, or favoring by the United States Government or any agency thereof. The views and opinions of authors expressed herein do not necessarily state or reflect those of the United States Government or any agency thereof. 


\section{DISCLAIMER}

Portions of this document may be illegible in electronic image products. Images are produced from the best available original document. 
Sändberg, D. Smith, R.F. Somodi, I. Stancu, W.D. Strossman, M. Sung, R. Tayloe, G.J. VanDalen, W. Vernon, N. Wadia, D.H. White, S. Yellin, H. Yi (LSND Collaboration) Phys. Rev. Lett. Volume 92, Number 9 (2004) 1774-1777hep-ex/0310060. I must emphasize that the entire idea and work for this paper were from Southern University and this PRL constitutes one only four PRL from the entire LSND collaboration.

We now briefly discuss the importance of this paper. The observation of the decay $\pi^{o} \rightarrow \nu \tilde{\nu}$ would imply new interesting physics. The pion has zero spin and odd intrinsic parity, that is $J^{P}=0^{-}$; it is represented by a wave function which has the space transformation properties under inversion and rotation of a pseudo-scalar. The pion decay with a hadronic state of $J^{P}=0^{-}$to vacuum, $J^{P}=0^{+}$can only be achieved by axial vector (A) or pseudo-scalar (P) operators. Momentum and angular momentum conservation require that the decay $\nu$ and $\bar{\nu}$ possess the same helicity. If Majorana neutrinos exist, then neutrinos are identical to anti-neutrinos, or if the neutrino is massive, then the neutrino will not always respect the observed rule. A- and P-interaction favor leptons with opposite and same helicity, respectively. Therefore, in A-interaction the un-favored helicity $-\beta$ is proportional to $(1-\beta)$ and in the $\mathrm{P}$-interaction the decay rate is proportional to $(1+\beta)$. This implies that A-interaction forbids $\pi^{\circ} \rightarrow \nu \bar{\nu}$ decay for massless neutrinos and that $\mathrm{P}$ coupling maximizes decay rate for neutrino with zero mass. If the neutrino mass is not zero and the $Z^{0}$ couples to the right-handed neutrino with standard weak-interaction strength, the branching ratio $B\left(\pi^{o} \rightarrow \nu \bar{\nu}\right)$ has a maximum value of $3 \times 10^{-9}$ at $m_{\nu}=55 \mathrm{MeV} / \mathrm{c}^{2}$; the upper limit $m_{\nu_{\tau}} \leq 18.2 \mathrm{MeV} / \mathrm{c}^{2}$ implies that $B\left(\pi^{\circ} \rightarrow \nu \bar{\nu}\right)<5.2 \times 10^{-10}$ [1]. It is noteworthy that a BR of $\approx 10^{-14}$ for the $\pi^{o} \rightarrow \nu \nu \gamma$ within the Standard Model is allowed.

This analysis was the topic for two MS theses here at Southern, Rodica Somodi and Hai Yi.

We supported these students with other funding sources. Some undergraduate students such as Billy J. Vegara (US Navy), Tho Phan, Michael Davis and Ali Sabahi were supported part-time using this grant. Two months of my summer salary was provided by this grant. We also had two other summer students, Valencia Walker and Kema Willians who worked on LSND but were supported by LANL through their Office of Diversity.

We believe that our contribution to LSND was substantial and we were very successful to fulfill our obligations with respect to our commitments to this contract.

We In conclusion, we received a total $\$ 251,000$ from DoE/HEP for the entire 9-year duration of this grant. This grant provided two months summer salary for myself, Ali $R$. Fazely, travel money and some undergraduate student support. We thank the DoE/HEP office for this grant and we hope to work with them in the future. 


\section{References}

[1] H. ALbrecht et. al., Phys.Lett. B 202, 149 (1988). 